\title{
Educación y Tecnologías; Herramientas Situadas en el Contexto Social
}

\author{
Yeimy Hernández Ricardo \\ yeimy.hernandez@seiem.edu.mx \\ Universidad Pedagógica Nacional Unidad 151, Sede Regional Ixtlahuaca, México. \\ https://orcid.org/0000-0003-0893-7722 \\ Patricia Camacho Barrios \\ patricia.camacho@seiem.edu.mx \\ Universidad Pedagógica Nacional Unidad 151, Sede Regional Ixtlahuaca, México. \\ https://orcid.org/0000-0002-8158-9923
}

Recepción: 06 de julio del 2021

Aprobación: 08 de agosto del 2020

Publicación: 06 de junio de 2021

\section{Resumen}

El presente artículo hace mención al uso de las tecnologías de la información y comunicación en educación desde los principales retos y muestra un panorama futuro de las TIC y su relación en el ámbito educativo. El inicio está plasmado por la definición, de lo que actualmente se llama sociedad del conocimiento y cómo su evolución, producto de la tecnología, ha abarcado distintas áreas, permitiendo innovar a la educación, estimulando la creación de nuevos conocimientos, de esta manera se describe la importancia de cada agente educativo (docente-alumno) y su rol transformador en el proceso de enseñanza-aprendizaje.

Palabras clave: TIC, tecnologías, información, comunicación.

\section{Education and Technologies; Tools Located in the Social Context}

Resume

This article makes mention of the use of information and communication technologies in education from the main challenges and shows a future panorama of ICT and its relationship in the educational field. The beginning is shaped by the definition of what is currently called the knowledge society and how its evolution, a product of technology, has covered different areas, allowing education to innovate, stimulating the creation of new knowledge, in this way it is 
described the importance of each educational agent (teacher-student) and their transforming role in the teaching-learning process.

Keywords: ICT, technologies, information, communication

\section{Introducción}

Abordar el tema de las tecnologías es abrir una puerta al debate en el que se están presentes en todos los niveles de nuestra sociedad actual desde las grandes corporaciones multinacionales, gobiernos, administraciones, universidades, centros educativos, organizaciones socioeconómicas y asociaciones profesionales y particulares.

Las personas somos seres sociales que requieren estar en constante comunicación e interacción, de ahí la importancia de incluir es uso de las TICS en el ámbito educativo, así como la manera en la que estas tienen impacto durante el proceso de enseñanza aprendizaje dentro y fuera de los centros destinados a la formación académica de los educandos.

Cada elemento de las tecnologías en el mundo globalizado, en el que nos encontramos es de suma importancia y permite integrarse a las nuevas normas bajo las que trabaja la educación. Si bien las tecnologías han abierto puertas importantes en el camino del ser humano, principalmente en el intercambio de ideas y diferentes tratados. Se hace una pausa y se analizan lo importante que son a diario, se observa que no solo son una herramienta educativa, sino que también permiten fortalecer el campo laboral y así mismo tener mayor acceso a información. Según la UNESCO (2013)

Contar con alfabetización digital básica, es hoy una necesidad no solo para lograr mejores procesos de aprendizaje de los estudiantes, sino también para tener más herramientas en el ámbito laboral y también para ejercer nuestra ciudadanía, pero es insuficiente si el acceso y la formación no posibilitan el desarrollo de usos innovadores y nuevas experiencias de aprendizaje (p. 20).

Revista RedCA, Vol. 4, Núm. 10 (junio-septiembre 2021) Universidad Autónoma del Estado de México, ISSN-e: 2594-2824 


\section{Las tecnologías y su uso cotidiano en la educación.}

Cada elemento tecnológico da pauta a lograr avances en los diferentes ámbitos educativos, científicos, políticos, económicos e incluso sociales. Sin embargo, surgen dos interrogantes importantes durante esta investigación ¿Qué nos ofrecen la tecnología?, ¿la tecnología realmente está al alcance de todos? Estas preguntas permitirán profundizar más en el tema y a su vez se llevará a cabo una reflexión sobre los alcances, así como el impacto que estas tienen en la vida del ser humana y el contexto en el que se desarrolla.

La tecnología es un elemento que ha tomado relevancia en estos últimos años, ya que se busca estar en constante comunicación con el mundo entero, siembre a la vanguardia de cualquier situación. Por ello Cabero (1998) hace referencia a que:

En líneas generales podríamos decir que las nuevas tecnologías de la información y comunicación son las que giran en torno a tres medios básicos: la informática, la microelectrónica y las telecomunicaciones; pero giran, no sólo de forma aislada, sino lo que es más significativo de manera interactiva e interconexionadas, lo que permite conseguir nuevas realidades comunicativas. (p. 198)

Si bien Cabero señala que las tecnologías van más allá de solo comunicar e informar, pues con ellas también se busca tele comunicarnos, hecho que en estos momentos asume gran relevancia, desde hace uno meses la sociedad se vio en la necesidad de retomar este aspecto como herramienta de aprendizaje y comunicación. El sistema federal opto por mantener informada a la sociedad por medio de esta herramienta y no solo eso, sino retomo aspectos educativos por medio de la televisión y redes sociales.

Cada día la sociedad se va enfrentando a situaciones diversas que exigen estar en constante cercanía con las tecnologías y siempre a la vanguardia ante los constantes retos ante ello A. Bautista y C. Alba, 1997 señalan que para Antonio Bartolomé:

"La T.E. encuentra su papel como una especialización dentro del ámbito de la Didáctica y de otras ciencias aplicadas de la Educación, refiriéndose especialmente 
al diseño, desarrollo y aplicación de recursos en procesos educativos, no únicamente en los procesos instructivos, sino también en aspectos relacionados con la Educación Social y otros campos educativos. Estos recursos se refieren, en general, especialmente a los recursos de carácter informático, audiovisual, tecnológicos, del tratamiento de la información y los que facilitan la comunicación” (p.2)

Apropiarse de las tecnologías es un reto que se debe enfrentar con responsabilidad y sobre todo con entusiasmo, pues existen situaciones en las que no se veía posible implementar estrategias tecnológicas, sin embargo, hoy nos damos cuenta que ante las necesidades que emergen de una sociedad globalizada esto ha sido necesario.

Bautista, A. y Alba, C. (1997) " en su obra ¿Qué es Tecnología Educativa? Comparten que Jordi Adell señala que se genera un cambio de ideas de acuerdo a las características y sobre todo a las nuevas posibilidades que ofertan las redes, por ello el autor menciona que:

El paradigma de las nuevas tecnologías son las redes informáticas. Los ordenadores, aislados, nos ofrecen una gran cantidad de posibilidades, pero conectados incrementan su funcionalidad en varios órdenes de magnitud. Formando redes, los ordenadores sirven [...] como herramienta para acceder a información, a recursos y servicios prestados por ordenadores remotos, como sistema de publicación y difusión de la información y como medio de comunicación entre seres humanos (p. 197).

El uso de las tecnologías multimedia e Internet hace posible el aprendizaje en prácticamente cualquier escenario (la escuela, la universidad, el hogar, el lugar de trabajo, los espacios de ocio, etc.), sin embargo, cabe señalar que existen espacios en los que difícilmente se tiene acceso a las mismas, lo que dificulta que los estudiantes puedan aprender por medio de estas.

\section{Integración de las TIC y la educación.}

El impacto de las TIC, dentro de la sociedad del conocimiento ha traído grandes cambios, respecto a forma y contenido, el efecto ha sido masivo y multiplicador, de tal forma que el sentido del conocimiento ha calado en la sociedad en general, y una de las grandes implicancias y 
modificaciones, es la educación. Parra (2012), menciona que uno de los lugares donde la tecnología ha influenciado mayoritariamente es en la escuela, y este a su vez en el oficio maestro, llegando a formar parte de la cotidianidad escolar.

La incorporación de las TIC, a la educación se ha convertido en un proceso, cuya implicancia, va mucho más allá de las herramientas tecnológicas que conforman el ambiente educativo, se habla de una construcción didáctica y la manera cómo se pueda construir y consolidar un aprendizaje significativo en base a la tecnología, en estricto pedagógico se habla del uso tecnológico a la educación. (Díaz-Barriga, 2013)

La transformación que ha sufrido las TIC, han logrado convertirse en instrumentos educativos, capaces de mejorar la calidad educativa del estudiante, revolucionando la forma en que se obtiene, se maneja y se interpreta la información. Aguilar (2012).

Dentro de los roles que asumen cada agente educativo, los estudiantes actuales, utilizan las herramientas tecnológicas para facilitar el aprendizaje; esta evolución surgió desde las primeras concepciones con la calculadora, el televisor, la grabadora, entre otras; sin embargo, el progreso ha sido tal que los recursos tecnológicos se han convertido en recursos educativos, donde la búsqueda por mejorar el aprendizaje trae consigo la tarea de involucrar la tecnología con la educación.

\section{Uso correcto de las tecnologías de la información en la educación.}

La incorporación de las TIC a la educación escolar que acabo de mencionar marcan un gradiente para la alfabetización digital. Esta se contempla habitualmente como el aprendizaje del uso funcional de las TIC, lo que lleva lógicamente a abordarla mediante la incorporación de los contenidos de aprendizaje al currículo escolar.

El logro de integrar las TIC en la educación, depende en gran medida de la habilidad del docente para estructurar el ambiente de aprendizaje (Unesco, 2008); mucho se habla, de dar el "salto" y "romper" esquemas tradicionales con un aprendizaje basado en la cooperación y el trabajo en equipo; sin embargo, el uso e involucramiento de las TIC en la educación, aún no ha sido entendido como aquella herramienta por la cual se pueda generar un aprendizaje significativo, errores 
frecuentes en la escuela reducen a las TIC a aquella herramienta que permite acceder y transmitir información, error que sigue englobando a la educación tradicional.

Este docente debe estructurar su función, organizando la forma en la cual los estudiantes adquieren competencias cognitivas y logren aplicarlas en situaciones diversas (Unesco, 2008). Las clases presenciales que se desarrollan en un aula, necesitarán de nuevos espacios que complementen el conocimiento mediante el uso de medios tecnológicos entre estudiantes y docentes, la aparición de las TIC encaja fácilmente en este proceso.

El estudiante participa como aquel nuevo agente educativo, quien producto de haber nacido en una sociedad tecnificada, se ha convertido en el elemento principal para la comunicación e interacción social. La diversidad de escenarios, contextos y tendencias en la educación, en la actualidad, imponen nuevos roles al proceso formativo, los que implican retos para el profesional del futuro y las instituciones y agentes encargados de su formación. Las TIC como herramientas añadidas a los modelos pedagógicos pueden convertirse en recursos valiosos para el aprendizaje, logrando formar estudiantes con competencias personales y profesionales idóneas para el desarrollo de un país.

La capacidad mediadora de las TIC como instrumentos psicológicos es una potencialidad que, como tal, se hace o no efectiva, y se hace efectiva, en mayor o menor medida, en las prácticas educativas que tienen lugar en las aulas en función de los usos que los participantes hacen de ellas. Cómo podemos identificar y describir estos usos, y de qué depende que los participantes en un proceso educativo determinado hagan unos u otros usos de las tecnologías disponibles, son dos preguntas que están actualmente en el punto de mira de un buen número de investigaciones y trabajos.

Por consiguiente se considera el recurso de la musicoterapia para el manejo de emociones, por que ayuda a una mejor convivencia humana por ejemplo la técnica el efecto Mozart que es la propiedad de algunos tonos y ritmos que ayuda a fortalecer la mente, a vivificar la creatividad, a activar emociones y a sanar el cuerpo (Campbell, 1998).

La música produce efectos beneficiosos en el sistema sensorial, cognitivo y motor (de forma sedante o estimulante), estimula la creatividad, el pensamiento, el leguaje, el aprendizaje y la memoria. Es un estímulo agradable y placentero para el cerebro que ayuda también a la relajación, efecto conocido desde la estimulación incluso dentro del útero materno o etapa prenatal.

Revista RedCA, Vol. 4, Núm. 10 (junio-septiembre 2021) Universidad Autónoma del Estado de México, ISSN-e: 2594-2824 
Asimismo, se tiene el propósito de exponer la utilidad práctica de esta estrategia para crear ambientes inclusivos, armónicos, libres de violencia, donde los alumnos puedan desarrollar sus capacidades, pensamiento crítico, a partir de sus habilidades, sociales y emocionales y la expresión asertiva de sus sentimientos. Si los alumnos en estas etapas iniciales de su educación, aprende a manejar adecuadamente sus emociones, van a lograr un mejor aprovechamiento de sus aprendizajes esperados, mejores relaciones colectivas, ambiente aprendizaje inclusivos, humanistas y con perspectiva intercultural.

Al trabajar en el manejo de las emociones, tendremos alumnos con una mejor salud mental para desarrollar al máximo sus habilidades, porque van a estar seguros y concentrados en el avance de las actividades y el máximo aprovechamiento de sus aprendizajes. En esta época de pandemia, es importante retomar las emociones de los alumnos por medio de la educación a distancia, implementar la músico terapia, como un medio de relajación, para olvidarse un poco de tanto estrés por este confinamiento en casa, mediante la implementación en las planeaciones.

Una actividad podría ser el taller de musicoterapia: revisar link https://youtu.be/5K6cTECVu2Y.

Las tecnologías de la información y la comunicación (TIC), se desarrollan a partir de los avances científicos producidos en los ámbitos de la informática y las telecomunicaciones. Es el conjunto de tecnologías que permiten el acceso, producción, tratamiento y comunicación de información presentada en diferentes códigos (texto, imagen, sonido, video).

El uso de los tics en los sistemas educativos es común debido a que son considerados una competencia básica, representa una oportunidad para el crecimiento económico y empleo y son herramientas para mejorar el proceso de enseñanza y aprendizaje. En el proceso de enseñanza puede mejorar los logros de aprendizaje de los alumnos, orienta a mejorar el currículum, la pedagogía, la evaluación, desarrollo profesional de profesor y otros aspectos de la cultura educativa, permite un nuevo escenario para la enseñanza y el aprendizaje y son una herramienta para aprender y para enseñar.

\section{Conclusiones}

Abordar el tema de las tecnologías es abrir una puerta al debate en el que se están presentes en todos los niveles de nuestra sociedad actual desde las grandes corporaciones multinacionales, 
gobiernos, administraciones, universidades, centros educativos, organizaciones socioeconómicas y asociaciones profesionales y particulares.

Gracias a la familiarización con las TICS, es como hay una nueva cultura donde predomina el ordenador sobre el libro o el docente y, actualmente ya no sólo sirve lo que los libros dicen, pues pueden aprender, cada vez más, por sí mismos, plantear, planificar estrategias y resolver situaciones en permanente transformación es gracias a su accesibilidad a los medios temáticos (Sánchez, 2009).

El logro de integrar las TIC en la educación, depende en gran medida de la habilidad del docente para estructurar el ambiente de aprendizaje (Unesco, 2008); mucho se habla, de dar el "salto" y "romper" esquemas tradicionales con un aprendizaje basado en la cooperación y el trabajo en equipo; sin embargo, el uso e involucramiento de las TIC en la educación, aún no ha sido entendido como aquella herramienta por la cual se pueda generar un aprendizaje significativo, errores frecuentes en la escuela reducen a las TIC a aquella herramienta que permite acceder y transmitir información, error que sigue englobando a la educación tradicional. (Mestres, 2008)

Este docente debe estructurar su función, organizando la forma en la cual los estudiantes adquieren competencias cognitivas y logren aplicarlas en situaciones diversas (Unesco, 2008). Las clases presenciales que se desarrollan en un aula, necesitarán de nuevos espacios que complementen el conocimiento mediante el uso de medios tecnológicos entre estudiantes y docentes, la aparición de las TIC encaja fácilmente en este proceso.

\section{Referencias}

Revista RedCA, Vol. 4, Núm. 10 (junio-septiembre 2021) Universidad Autónoma del Estado de México, ISSN-e: 2594-2824 
Aguilar, M. (2012). Aprendizaje y Tecnologías de Información y Comunicación: Hacia nuevos escenarios educativos. Revista Latinoamericana de Ciencias Sociales, Niñez y Juventud, $10(2), 801-811$

Cabero, J. (1998) Impacto de las nuevas tecnologías de la información y la comunicación en las organizaciones educativas. En Lorenzo, M. y otros (coords): Enfoques en la organización y dirección de instituciones educativas formales y no formales (pp. 197-206). Granada: Grupo Editorial Universitario.

Campbell, D. (1998). El efecto Mozart: aprovechar el poder de la música para sanar el cuerpo, fortalecer la mente y liberar el espíritu creativo I. Barcelona.

Díaz-Barriga, F. (2013). TIC en el trabajo del aula. Impacto en la planeación didáctica. Revista Iberoamericana de Educación Superior, 4(10), 3-21. Doi: 10.1016/S2007-2872(13)719218

Oficina Regional de Educación para América Latina y el Caribe UNESCO Santiago. (2013). Enfoques estratégicos sobre las TICS en educación en América Latina y el Caribe. UNESCO: Chile.Bautista, A. y Alba, C. (1997) "¿Qué es Tecnología Educativa?: Autores y significados", Revista Píxel-bit, $\mathrm{n}^{\mathrm{o}} \quad 9$, 4.. Recuperado de http://www.us.es/pixelbit/art94.htm

Sánchez Asín, Antonio y Boix Peinado, Josep Lluis (2009). La Sociedad del Conocimiento y las TIC: Una inmejorable oportunidad para el cambio docente. Pixel - Bit, Revista de Medios y Educación, N³4, pp. 179 - 204, España. 